\title{
Periodicity Analysis of Mrk 501 and Mrk 421 in Gamma
} Rays

\author{
Roman lotov ${ }^{a, *}$ A. Arbet-Engels, ${ }^{b}$ P. Arras,${ }^{c}$ D. Baack,${ }^{d}$ M. Balbo,${ }^{e}$ A. Biland,${ }^{b}$ M. \\ Böttcher, ${ }^{g}$ T. Bretz, ${ }^{c, f}$ J. Buss, ${ }^{d}$ D. Dorner, ${ }^{a}$ V. Eberle, ${ }^{g}$ L. Eisenberger, ${ }^{a}$ D. \\ Elsaesser, ${ }^{d}$ T. Enßlin, ${ }^{c}$ D. Hildebrand, ${ }^{b}$ A. Kalenski, ${ }^{a}$ A. Kostic ${ }^{c}$ M. Kreter, ${ }^{g}$ K. \\ Mannheim, ${ }^{a}$ V. Marchenko, ${ }^{h}$ A. Mitchell, ${ }^{b}$ D. Neise, ${ }^{b}$ M. Noethe, ${ }^{d}$ A. Paravac,${ }^{a}$ W. \\ Rhode ${ }^{d}$ B. Schleicher, ${ }^{a}$ V. Sliusar, ${ }^{e}$ M. Tarnopolski, ${ }^{h}$ F. Theissen, ${ }^{c}$ R. Walter ${ }^{e}$ and N. \\ Żywucka ${ }^{g}$ \\ ${ }^{a}$ Universität Würzburg, Lehrstuhl für Astronomie, Würzburg Germany \\ ${ }^{b}$ ETH Zurich, Institute for Particle Physics and Astrophysics, Otto-Stern-Weg 5, 8093 Zurich, Switzerland \\ ${ }^{c}$ Max-Planck-Institut für Astrophysik, Karl-Schwarzschild-Straße 1, 85748 Garching, Germany \\ ${ }^{d}$ TU Dortmund, Experimental Physics 5, Otto-Hahn-Str. 4a, 44227 Dortmund, Germany \\ ${ }^{e}$ University of Geneva, Department of Astronomy, Chemin d'Ecogia 16, 1290 Versoix, Switzerland \\ ${ }^{f}$ III. Physikalisches Institut A, RWTH Aachen University, Templergraben 55, 52062 Aachen, Germany \\ ${ }^{g}$ Centre of Space Research, North-West University, Potchefstroom 2520, South Africa \\ ${ }^{h}$ Astronomical Observatory, Jagiellonian University, Orla 171, 30-244, Kraków, Poland \\ E-mail: roman.iotov@stud-mail.uni-wuerzburg.de
}

Blazars, a subclass of active galactic nuclei (AGNs), are highly variable objects. Quasiperiodic oscillations (QPOs), which might originate from a binary black hole located at the AGN core, have been found in some blazar light curves. For the blazars Mrk 421 and Mrk 501, we test the possible QPO behavior using a variety of methods (generalized Lomb-Scargle periodogram, continuous-time autoregressive moving average models, wavelet scalograms, and $\mathcal{A}-\mathcal{T}$ plane), studying systematic effects in detail. We use gamma-ray light curves from FACT, a ground-based imaging air Cherenkov telescope. We report herein on a $3 \sigma$-significant QPO in Mrk 421.

$37^{\text {th }}$ International Cosmic Ray Conference (ICRC 2021)

July 12 th - 23rd, 2021

Online - Berlin, Germany

\footnotetext{
${ }^{*}$ Presenter
} 


\section{Introduction}

Previous studies on the periodicity in Mrk 421 and Mrk 501 arrived at conflicting results.

For Mrk 421, Li et al. [10] performed a Lomb-Scargle analysis of $15 \mathrm{GHz}$, X-ray, and gammaray light curves. They found hints of a $283 \mathrm{~d}$ periodicity in $15 \mathrm{GHz}$ and gamma rays, and a $309 \mathrm{~d}$ periodicity in X-rays. The authors argued for this to be evidence of a binary black hole (BBH) system. Using the spectral analysis tool RobPer, Benitez et al. [8] also reported on a periodicity of $310 \mathrm{~d}$ in optical, X-ray, and gamma-ray data, supporting the BBH hypothesis as well. Bhatta and Dhital [9] found a $280 \mathrm{~d}$ periodicity in gamma rays, and Nilsson et al. [11] found a period of $477 \mathrm{~d}$ in the optical. Sandrinelli et al. [7] analyzed gamma-ray and optical light curves and, contrary to previous findings, found no significant periodicity in either. Likewise, Tarnopolski et al. [20] detected no QPOs in the gamma-ray light curve from Fermi-LAT.

For Mrk 501, there are also several papers that claim to have detected periodicities. Rieger and Mannheim [14] reported on a period of $23 \mathrm{~d}$ during a flare. Rödig et al. [15] analyzed several X-ray and gamma-ray light curves and detected a $72 \mathrm{~d}$ periodicity that they argued was possibly a higher harmonic of the $23 \mathrm{~d}$ QPO. Wang et al. [13] analyzed X-ray data from the RXTE satellite and found a period of $630 \mathrm{~d}$. Bhatta [6] claimed a $332 \mathrm{~d}$ periodicity in the Fermi-LAT light curve which was, however, not confirmed later on by Tarnopolski et al. [20]. Beck et al. [16] analyzed FACT data and found a hint of a periodicity at $332 \mathrm{~d}$ using the Lomb-Scargle periodogram (LSP).

These previous results are not always in agreement, and FACT, though it provides long monitoring of the two blazars, has been analyzed only once with the intent to search for QPOs [16]. We are therefore aiming at a systematic analysis of gamma-ray data including FACT and Fermi-LAT light curves utilizing a variety of methods to determine whether Mrk 421 and Mrk 501 show significant QPOs. I focus hereinafter on FACT data.

\section{Lomb-Scargle periodogram}

The LSP, introduced in [1], is a common tool for frequency analysis of unevenly sampled time series. Lomb [2] showed that the LSP is identical to a least squares spectral analysis at each frequency. Zechmeister and Kürster [3] extended it to the generalized LSP (GLSP) by adding a variable offset to the fitted sine functions. As described by VanderPlas [4], the GLSP can deal better with offsets in the data, but still suffers from some problems. Gaps in the data can be expressed as window functions, as well as the start and end times of data taking. These broaden any peak in the periodogram. Furthermore, periodic systematic influences on the data can also produce "false" periodicities. In case of FACT data, these are yearly changes, like the zenith distance of a source, and yearly gaps due to observability, as FACT is a ground-based telescope. Weather phenomena like dust are also quasiyearly influences. The FACT light curves for both blazars start in 2012 and end in 2019, data quality and cuts are further described in [16] and [29]. Furthermore, monthly gaps due to Moon light are present, but less problematic as most findings for these objects report periods outside this numbers. All of the mentioned influences can result in yearly and monthly peaks in the periodograms. A more problematic influence on the data is colored noise. 


\subsection{Influence of colored noise on LSP}

Blazars such as Mrk 421 and Mrk 501 are very variable objects that exhibit colored-noise power spectral densities (PSDs). Colored noise is a form of correlated noise that produces larger changes in flux amplitudes on longer time scales. Its power spectral density (PSD) is described by a power law (PL):

$$
P(f) \propto f^{-\alpha},
$$

where $\alpha$ is the PL index. Colored noise produces higher peaks at higher periods in the LSP [5]. This can be understood as the flux amplitude changing due to the variability caused by the colored noise. These changes will be larger for longer time intervals. Thus at large periods in the light curve, these flux variations will be detected by the LSP as oscillations. This complicates the interpretation of the periodogram, as QPOs are mostly reported at large periods. Furthermore, estimating the significance of a peak in the periodogram can not be easily done analytically, as the false alarm probability usually assumes white noise (i.e., uncorrelated) as the null hypothesis [4].

\subsection{Simulation of light curves}

In order to assess the significance of the LSP peaks, we choose to numerically generate artificial light curves. For this, we follow Emmanoulopoulos et al. [17] who provide a method to produce light curves with a given probability density function (PDF) and PSD.

The flux $x$ distributions are fitted with a mixture of a normal distribution and a log-normal distribution with weights $w$ and $(1-w)$ :

$$
\begin{gathered}
f(x)=w f_{\text {norm }}(x)+(1-w) f_{\text {log }}(x), \\
f_{\text {norm }}(x)=\frac{1}{\sigma \sqrt{2 \pi}} \exp \left(-\frac{(x-\mu)^{2}}{2 \sigma^{2}}\right), \\
f_{\text {log }}(x)=\frac{1}{(x-s) \sigma \sqrt{2 \pi}} \exp \left(-\frac{\left(\ln ^{2}(x-s)-\mu\right)^{2}}{2 \sigma^{2}}\right),
\end{gathered}
$$

where the $f_{l o g}$ includes an additional shift $s$ to account for the negative values present in the FACT data. The fits were obtained by maximizing the log-likelihood numerically with the scipy.optimize module. For Mrk 421, we obtain $\mu_{l o g}=-0.284, \sigma_{l o g}=0.631, s=-0.175$ for the log-normal distribution, and $\mu_{\text {norm }}=0.193, \sigma_{\text {norm }}=0.368$ for the normal component, with $w=0.168$. For Mrk 501, we obtain $\mu_{\text {log }}=0.319, \sigma_{\text {log }}=0.405, s=-0.98$ for the lognormal distribution, and $\mu_{\text {norm }}=0.086, \sigma_{\text {norm }}=0.12$ for the normal component, with $w=0.476$. Histograms and the fitted PDFs are shown in Figure 1.

To model the colored noise we follow [18]. We compute the LSP of the FACT data on an evenfrequency grid of $10^{4}$ points between the pseudo-Nuyquist frequency of $1 / 2 \mathrm{~d}$ and the minimum frequency of $1 / 1000 \mathrm{~d}$. This constitutes an oversampling by a factor of $n_{0}=5$, consistent with the recommendation to use $n_{0}=5-10$ [4]. Since the frequency grid is evenly spaced, in order for the higher frequencies not to dominate the PL fit when working in the log-log space, we bin the periodograms accordingly. The binning is chosen to be 15 equally spaced bins in the log-frequency space, between the maximum frequency and the pseudo-Nuyquist frequency. The power in a bin 


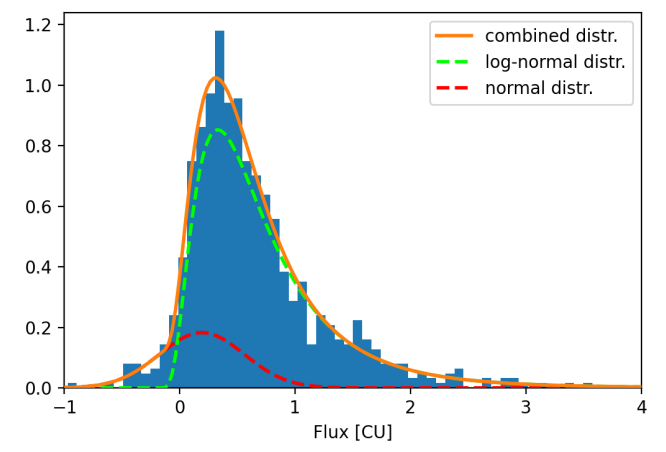

(a) Mrk 421

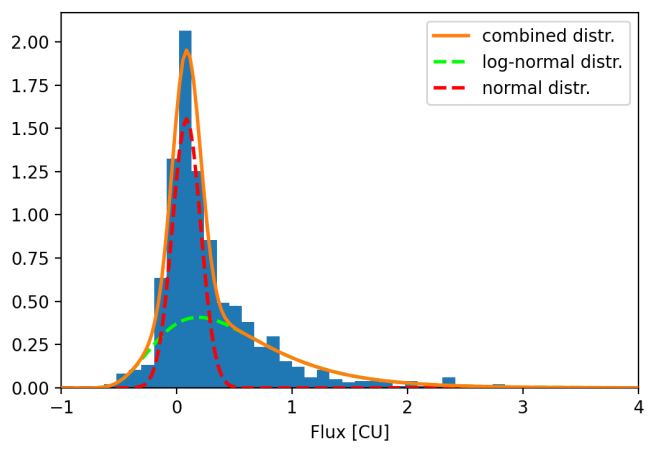

(b) Mrk 501

Figure 1: Flux distributions in Crab units [CU], with weighted log-normal and normal mixture fits.

is calculated as the arithmetic mean of the logarithmized powers. The binned PSDs are then fitted with a linear model in the log-log space to obtain the PL index as slope. Results are displayed in Figure 2

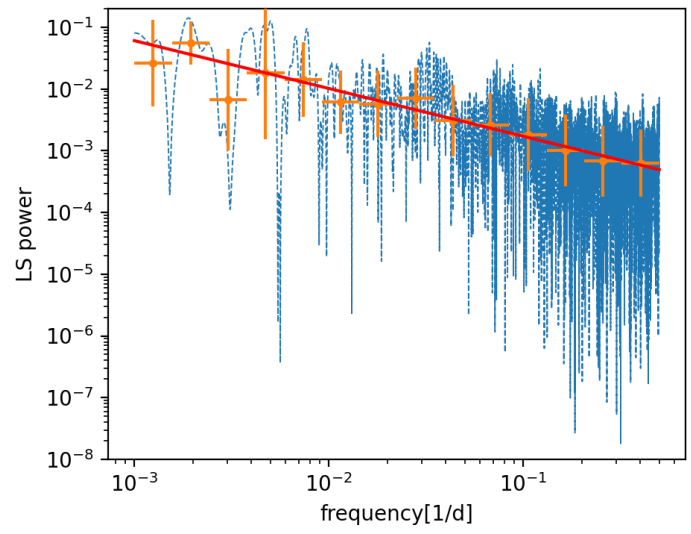

(a) Mrk 421

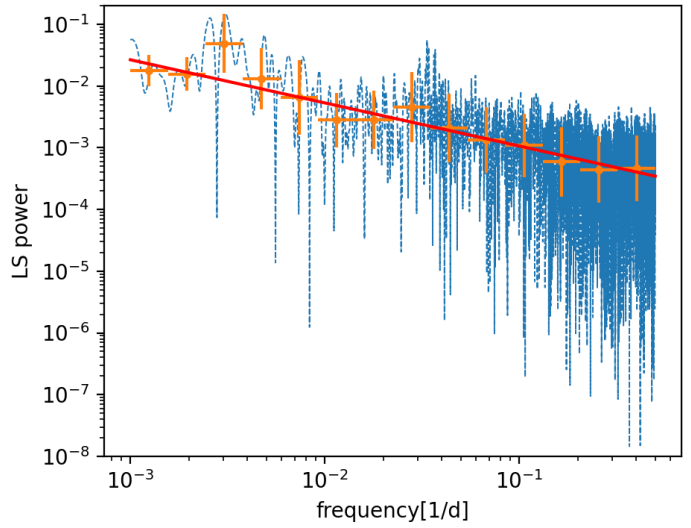

(b) Mrk 501

Figure 2: Lomb-Scargle periodograms with linear fit in log-log space.

We obtain PL indices of $\alpha_{501}=0.699 \pm 0.064$ and $\alpha_{421}=0.774 \pm 0.061$. It is important to note that these are not the actual power law indices $\alpha^{\prime}$ of the data, but are lower due to leakage of colored noise. This is due to the observational gaps in the data. As colored noise and the PL index describe the correlation of noise in the data, gaps and thereby missing data lower the measured slope.

In order to compensate for this, we simulate light curves with higher colored noise indices $\alpha^{\prime}$ and then apply the same window functions that are present in the actual light curves. For light curve generation, we use the python script by Connolly [19] that implements the Emmanoulopoulos et al. [17] method of light curve generation. The determination of this $\alpha^{\prime}$ is again done numerically, by generating ensembles of $10^{3}$ light curves per $\alpha^{\prime}$ in increments of 0.01 and applying the window functions on them. Afterwards, for each light curve, an LSP is computed and the PSD slope is 
determined as described above. After determining the needed PL index $\alpha^{\prime}$, we can generate the final set of $10^{4}$ light curves to estimate the significance of the LSP peaks with the same PSD and PDF as in the data. For each generated light curve, an LSP is computed. From this set of periodograms, the significance can be directly determined by the percentage of light curves that reach a certain value in the periodogram. These results will be published in our upcoming paper.

\section{Further methods for periodicity analysis}

In order to determine whether Mrk 421 and Mrk 501 exhibit QPOs, we also employ other methods for periodicity analysis and PSD modeling. In the following, we give a short overview of the methods that will be used in our future publication, that are also described in detail by Tarnopolski et al. [20] and Selig et al. [27].

\subsection{Wavelet scalogram}

Unlike the LSP, which describes oscillations during the whole time series as sine waves, a wavelet scalogram is a decomposition of the time series into wavelets, i.e., short localized oscillations. Through a continuous wavelet transform a signal can be decomposed into a combination of wavelets. As such, the wavelet scalogram not only provides a global power, but also describes at which times periodic signals are present in the data. This can give additional insights into the origin of QPOs, for instance, if they are present more often during flaring activity or the quiescent states. We employ the Welch overlapping segment averaging method, used for irregularly sampled time series, as implemented in the python package WAVEPAL ${ }^{1}$ [22]. In Figures 3 and 4, scalograms for Mrk 421 and Mrk 501 are shown, respectively. Mrk 501 exhibits no periodicity, and a $3 \sigma$-significance period is present in Mrk 421 .

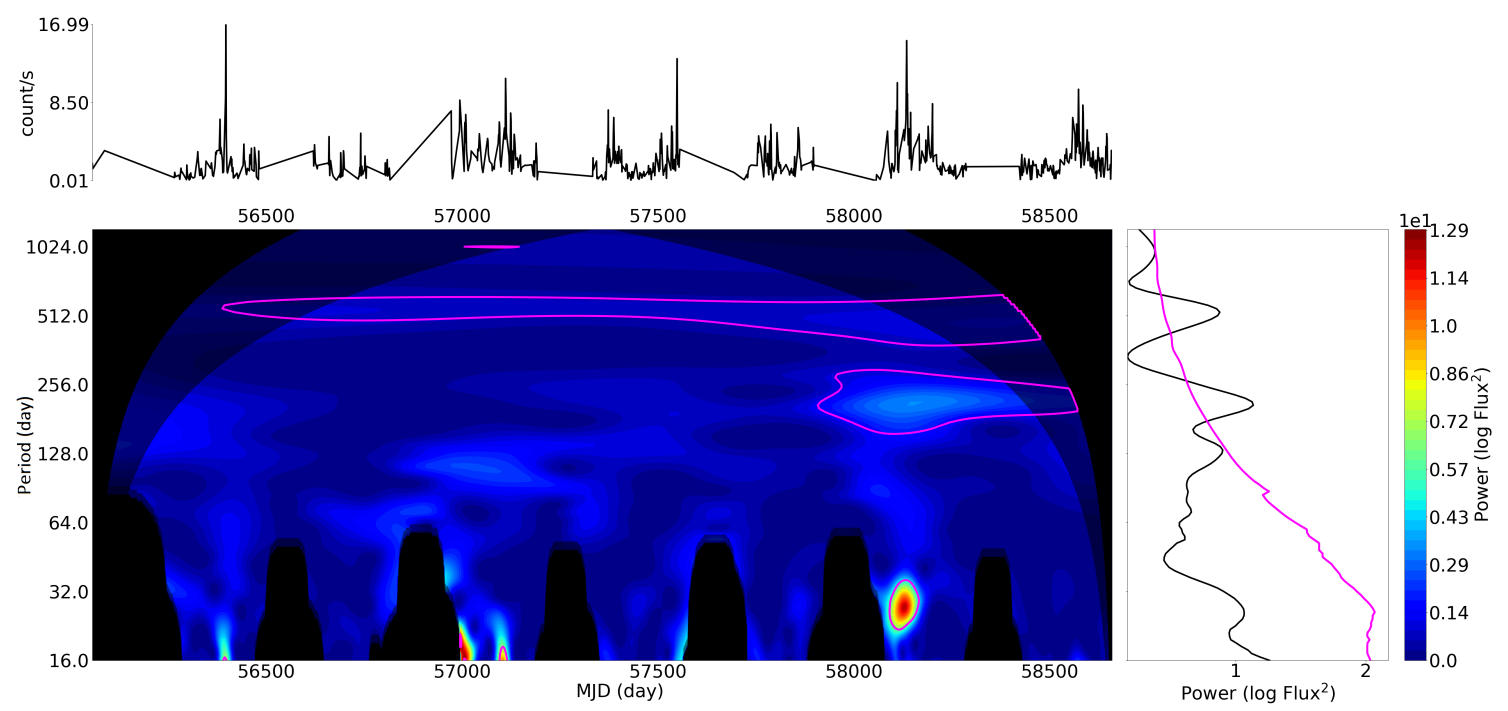

Figure 3: Wavelet scalogram of Mrk 421 in nightly binning. Pink line indicates a $3 \sigma$ level.

\footnotetext{
1https://github.com/guillaumelenoir/WAVEPAL
} 


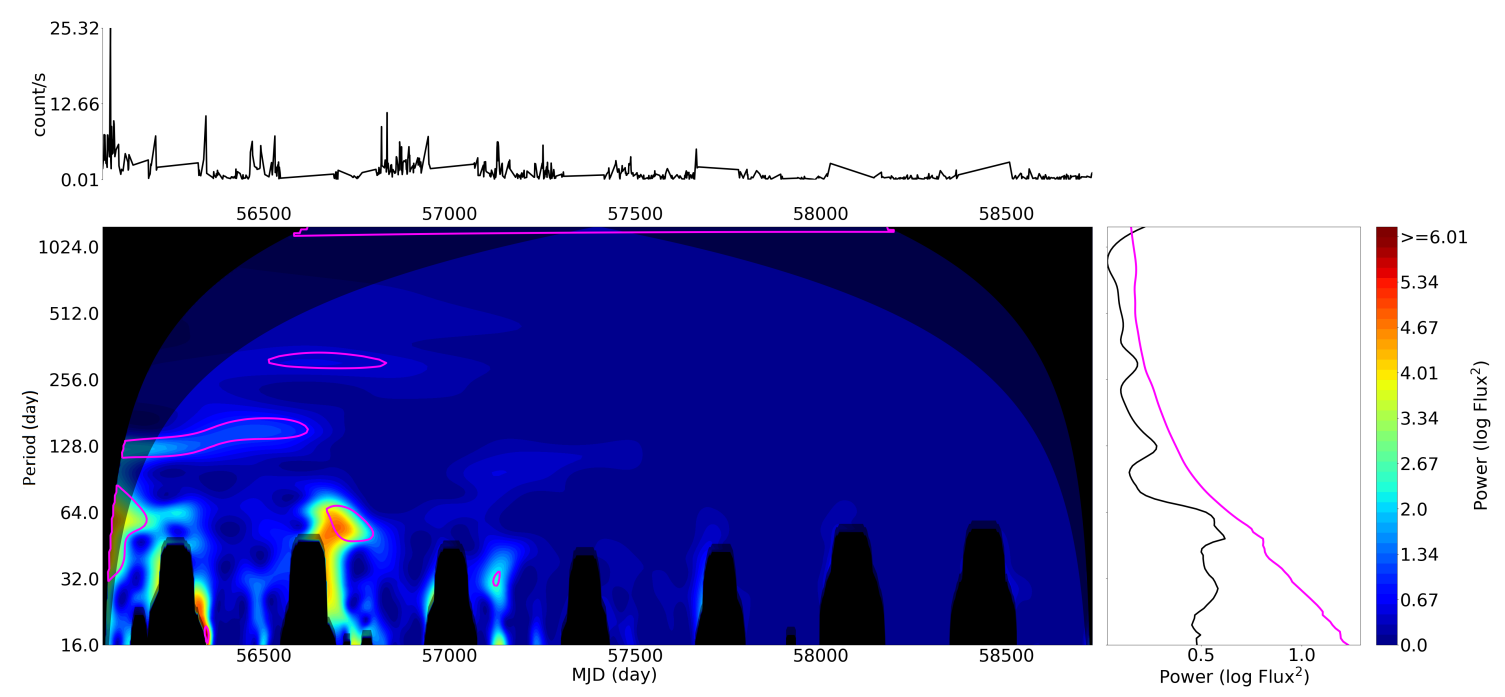

Figure 4: Wavelet scalogram of Mrk 501 in nightly binning. Pink line indicates a $3 \sigma$ level.

\section{$3.2 \mathcal{A}-\mathcal{T}$ plane}

The $\mathcal{A}-\mathcal{T}$ plane, first described by Tarnopolski [23] and further employed to classify blazar [20] and blazar candidates' [21] light curves, was introduced as a method to estimate the Hurst exponent (a measure of persistence, or long-term memory). The $\mathcal{A}-\mathcal{T}$ plane is spanned by the fraction of turning points $\mathcal{T}$ and the Abbe value $\mathcal{A}$. It is able to differentiate between different types of colored noise, characterized by different PL indices.

\subsection{CARMA}

The continuous-time autoregressive moving average (CARMA) stochastic process is described in detail in [24]. It models the light curves with a zero-mean Gaussian process, via a stochastic differential equation with autoregressive (AR) and moving average (MA) components. From this model a PSD can be determined analytically, can indicate the presence of QPOs, and differentiate them from noise. For this modeling, we use the publicly available MCMC sampler within carma_pack ${ }^{2}$ that was developed by Kelly et al. [25] for performing Bayesian inference of CARMA models.

\subsection{NIFTy}

Numerical information field theory (NIFTy), described by Selig et al. [27], is a new method for signal inference algorithms that utilizes information field theory. It was shown in [26] to be able to detect periodic signals from PG $1553+113$ in Fermi-LAT data, that were also previously detected using other methods by Ackermann et al. [28]. We will use this new method on FACT and Fermi-LAT data to further study the existence and significance of QPOs in Mrk 421 and Mrk 501.

${ }^{2}$ https://github.com/brandonckelly/carma_pack 


\section{Conclusions}

We present the current status of our search for QPOs in Mrk 421 and Mrk 501. For the LSP, we model the colored noise as well as the flux distribution based on the data to generate artificial light curves with the same observational gaps, noise, distributions of observables. These light curves are then used to estimate the significance of QPOs numerically. We furthermore employ the wavelet scalogram, detecting a $3 \sigma$-significant signal in Mrk 421, but not in Mrk 501. The final results, including using the $\mathcal{A}-\mathcal{T}$ plane, CARMA, and additional methods, utilizing Fermi-LAT light curves as well as, will be published in our forthcoming paper.

Acknowledgements The important contributions from ETH Zurich grants ETH-10.08-2 and ETH-27.12-1 as well as the funding by the Swiss SNF and the German BMBF (Verbundforschung Astro- und Astroteilchenphysik) and HAP (Helmoltz Alliance for Astroparticle Physics) are gratefully acknowledged. Part of this work is supported by Deutsche Forschungsgemeinschaft (DFG) within the Collaborative Research Center SFB 876 "Providing Information by ResourceConstrained Analysis", project C3. We are thankful for the very valuable contributions from E. Lorenz, D. Renker and G. Viertel during the early phase of the project. We thank the Instituto de Astrofísica de Canarias for allowing us to operate the telescope at the Observatorio del Roque de los Muchachos in La Palma, the Max-Planck-Institut für Physik for providing us with the mount of the former HEGRA CT3 telescope, and the MAGIC collaboration for their support.

\section{References}

[1] Scargle, J. D. 1982, ApJ, 263, 835

[2] Lomb, N. R. 1976, Ap\&SS, 39, 447

[3] Zechmeister, M. \& Kürster, M. 2009, A\&A 496, 577

[4] VanderPlas, J. T. 2018, ApJS 236, 16

[5] Vaughan, S. 2005, A\&A 431, 391

[6] Bhatta, G. 2019, MNRAS 487, 3990

[7] Sandrinelli, A., Covino, S., Treves, A. et al. 2017, A\&A, 600, A132

[8] Benitez, E., Cabrera J. I., Fraija, N. et al. 2015, arXiv:1512.01219

[9] Bhatta, G. \& Dhital, N. 2020, ApJ 891, 129

[10] Li, H. Z., Jiang, Y. G., Guo, D. F., Chen, X. \& Yi, T. F. 2016, PASP 128, 074101

[11] Nilsson, K., Lindfors, E., Takalo, L. O. et al. 2018, A\&A 620, A185

[12] Covino, S., Sandrinelli, A. \& Treves, A. 2019, MNRAS 482, 1270

[13] Wang, H., Yin, C. \& Xiang, F. 2017, ApSS 362, 99

[14] Rieger, F. M. \& Mannheim, K. 2000, A\&A 359, 948

[15] Rödig, C., Burkart, T., Elbracht, O. \& Spanier, F. 2009, A\&A, 501, 925

[16] Beck, M., Arbet-Engels, A., Baack, D. et al. 2019, 36th International Cosmic Ray Conference (ICRC2019), 36, 630

[17] Emmanoulopoulos, D., McHardy, I. M. \& Papadakis, I. E. 2013, MNRAS, 433, 907 
[18] Vaughan, S. 2005, A\&A, 431, 391

[19] Connolly, S. D. 2016, Astrophysics Source Code Library, record ascl:1602.012

[20] Tarnopolski, M., Żywucka, N., Marchenko, V. \& Pascual-Granado, J. 2020, ApJS, 250, 1

[21] Żywucka, N., Tarnopolski, M., Böttcher, M., Stawarz, Ł. \& Marchenko, V. 2020, ApJ, 888, 107

[22] Lenoir, G. \& Crucifix, M. 2018, Nonlin. Processes Geophys., 25, 175

[23] Tarnopolski, M. 2016, Phys A, 461, 662

[24] Moreno, J., Vogeley, M. S., Richards, G. T. \& Yu, W. 2019, PASP, 131, 063001

[25] Kelly, B. C., Becker, A. C., Sobolewska, M., Siemiginowska, A. \& Uttley, P. 2014, ApJ, 788, 33

[26] Kreter, M. 2019, Proceedings of the International Astronomical Union, 15(S356), 369

[27] Selig, M., Bell, M. R., Jurklewitz, H., et al. 2013, A\&A, 554, A26

[28] Ackermann, M., Ajello, M., Albert, A. et al. 2015, ApJ, 813, L41

[29] Arbet-Engels, A., Baack, D., Balbo, M. et al. 2021, A\&A, 647, A88 